\title{
Expériences de prise en charge du poids chez des adultes canadiens faisant de l'embonpoint ou obèses : résultats d'un sondage national
}

\author{
S. F. Kirk, Ph. D. (1); R. Tytus, M.D. (2); R. T. Tsuyuki, M.D. (3); A. M. Sharma, M.D. (3)
}

Cet article a fait l'objet d'une évaluation par les pairs.

\section{Résumé}

Introduction : Nous en savons peu sur le lien entre les Lignes directrices canadiennes de 2006 sur la prise en charge et la prévention de l’obésité et les expériences de prise en charge du poids des Canadiens, et nous ne savons pas si ces expériences cadrent avec les recommandations figurant dans les Lignes directrices.

Méthodologie : Nous avons utilisé les données d’un sondage omnibus auprès de la population générale pour comprendre ces deux enjeux, particulièrement en relation avec les maladies chroniques. Le sondage était composé de 23 questions sur les pratiques de prise en charge du poids et de questions sur les caractéristiques personnelles.

Résultats : Sur les 2004 répondants, 33 \% ont été classés comme faisant de l'embonpoint et $20 \%$ comme étant obèses. Parmi les répondants ayant de l'embonpoint ou obèses, $48 \%$ ont déclaré avoir interrogé leur médecin au sujet de la perte de poids et $30 \%$ ont affirmé que leur médecin leur avait suggéré de perdre du poids sans qu'ils aient eux-mêmes abordé la question. En rapport avec les recommandations figurant dans les Lignes directrices, $14 \%$ des personnes ayant de l'embonpoint et $18 \%$ de celles étant obèses ont dit que la circonférence de leur taille avait été mesurée, respectivement $82 \%$ et $87 \%$ ont affirmé que leur pression artérielle avant été vérifiée et respectivement $36 \%$ et $50 \%$ ont déclaré qu'elles avaient subi un test de dépistage du diabète.

Conclusion : Ces résultats ont une incidence sur le diagnostic et la prise en charge des maladies chroniques.

Mots-clés : embonpoint, obésité, lignes directrices de pratique clinique, diabète, hypertension, maladies chroniques

\section{Introduction}

$\mathrm{Au}$ cours des dernières décennies, les taux d'obésité ont augmenté de façon considérable, tant au Canada que dans les autres pays développés ${ }^{1,2}$. Étant donné que l'obésité est étroitement liée à un certain nombre de maladies chroniques, comme le diabète, les coronopathies et l'hypertension ${ }^{1}$, le nombre de cas observés de ces maladies est à la hausse. Par conséquent, la viabilité du système de soins de santé canadien est durement touchée $e^{3}$. Dans une analyse récente, on estimait à 6 milliards de dollars les coûts totaux directs attribuables à l'embonpoint et à l'obésité, ce qui représente $4,1 \%$ des dépenses en santé au Canada ${ }^{3}$.
Les Canadiens dont l'indice de masse corporelle (IMC) est supérieur à $30 \mathrm{~kg} / \mathrm{m}^{2}$ sont 4 fois plus susceptibles de souffrir du diabète, 3,3 fois plus susceptibles de présenter une hypertension artérielle et 1,5 fois plus susceptibles d'avoir une coronopathie $^{1}$. Une étude américaine récente suggère qu'en raison de l'augmentation marquée de la proportion de personnes obèses, l'obésité contribue désormais autant, sinon davantage, au fardeau des maladies que le tabagisme ${ }^{4}$. En outre, en comparaison avec les taux de référence, les taux de prévalence de l'obésité de classe II (IMC $35-39,9 \mathrm{~kg} / \mathrm{m}^{2}$ ) et de l'obésité de classe III (IMC $\geq 40 \mathrm{~kg} / \mathrm{m}^{2}$ ) sont ceux qui sont en plus forte hausse au Canada ${ }^{5}$. En d'autres termes, les personnes ayant de l'embonpoint ou obèses - soit celles qui sont le plus à risque de présenter les états comorbides énoncés plus haut - deviennent plus lourdes, plus vite ${ }^{4}$.

Malgré ces statistiques saisissantes, l’obésité n'est pas bien prise en charge par le système de santé actuel, une situation qui n'est pas propre au Canada ${ }^{6,7}$. Peu de professionnels de la santé donnent à leurs patients des conseils sur la prise en charge de leur poids en général ni ne fournissent de services de prise en charge de l'obésité ${ }^{8}$. En effet, un grand nombre de professionnels de la santé canadiens n'abordent même pas la question de l'obésité avec leurs patients ${ }^{9}$. Dans ce contexte, la publication des Lignes directrices canadiennes de 2006 sur la prise en charge et la prévention de l'obésité chez les adultes et les enfants a constitué un jalon

Rattachement des auteurs :

1. Dalhousie University, Halifax (Nouvelle-Écosse), Canada

2. McMaster University, Hamilton (Ontario), Canada

3. University of Alberta, Edmonton (Alberta), Canada

Correspondance : Sara Kirk, Chaire de recherche du Canada en recherche sur les services de santé, School of Health and Human Performance, Université Dalhousie, 1318 , rue Robie, Halifax (Nouvelle-Écosse) B3H 3E2; tél. : 902-494-8440; téléc. : 902-494-7567; courriel : sara.kirk@dal.ca 
important dans la lutte contre l'obésité ${ }^{10}$. Le but de ces Lignes directrices est d'entraîner des changements dans la pratique clinique et, en fin de compte, de diminuer le taux de prévalence de l'obésité et de ses complications chez les Canadiens ${ }^{11}$. Elles renferment 72 recommandations à l'appui de la prise en charge de l'obésité dans une vaste gamme de contextes, en insistant sur le rôle des professionnels de la santé au sein du système de soins de santé canadien.

À l'heure actuelle, nous en savons peu sur les expériences des Canadiens en matière de prise en charge du poids, pas plus que nous ne savons si leurs expériences s'inspirent des recommandations figurant dans les Lignes directrices. Une compréhension approfondie des expériences de prise en charge de l'embonpoint et de l'obésité pourrait permettre aux personnes souffrant de problèmes de poids d'obtenir le soutien approprié. En outre, l'analyse des expériences de ces personnes par rapport aux recommandations des Lignes directrices pourrait permettre de mieux connaître les pratiques de prise en charge du poids des professionnels de la santé et d'améliorer la façon dont ces derniers mettent les Lignes directrices en application. Compte tenu de leur grand nombre, il est impossible d'évaluer toutes les recommandations dans la pratique clinique courante. Trois d'entre elles sont particulièrement pertinentes sur le plan du diagnostic et de la prise en charge des maladies chroniques ${ }^{10}$ : la recommandation $n^{\circ} 4$, qui recommande la mesure de la circonférence de la taille chez tous les adultes afin d'évaluer les risques pour la santé reliés à l'obésité, et les recommandations $\mathrm{n}^{\text {os }} 6$ et 7 , qui insistent sur la nécessité de dépister les risques pour la santé et les complications connexes reliés à l'obésité ${ }^{10}$. Les pratiques de prise en charge du poids adoptées par les personnes faisant de l'embonpoint et les personnes obèses en relation avec ces trois recommandations servent de mesure substitutive de la mise en application des lignes directrices par les professionnels de la santé.

\section{Méthodologie}

Le sondage visait à déterminer les expériences de prise en charge du poids des adultes canadiens, c'est-à-dire à savoir
(1) si les personnes ayant de l'embonpoint ou obèses avaient cherché à obtenir de l'aide auprès d'un médecin de famille ou d'un autre professionnel de la santé relativement à la prise en charge de leur poids et, le cas échéant, (2) si leurs expériences de prise en charge du poids cadraient avec les trois recommandations retenues dans les Lignes directrices, à savoir la mesure de la circonférence de la taille (recommandation $n^{\circ} 4$ ) et le dépistage des états comorbides reliés au poids, comme l'hypertension et le diabète (recommandations $\mathrm{n}^{\text {os }} 6$ et 7 ).

Au nom du Réseau canadien en obésité, un organisme financé par le gouvernement fédéral, la firme Ipsos Reid a mené un sondage omnibus auprès de la population générale pendant deux semaines consécutives, du 23 mars au 3 avril 2009. Chaque semaine de sondage a permis d'obtenir environ 1000 réponses d'adultes canadiens. Le questionnaire a été rédigé par un groupe d'experts indépendants; il était composé de 23 questions sur les pratiques de prise en charge du poids, ainsi que de questions sur les caractéristiques personnelles (taille et poids autodéclarés, sexe, âge, revenu et région de résidence). L’approbation déontologique a été donnée par le Canadian SHIELD Ethics Review Board. La taille de l'échantillon a été choisie de façon à obtenir des données solides pour chaque groupe représentatif de la population en fonction de critères clés (p. ex. sexe, âge et lieu de résidence) $)^{12}$.

Le sondage a été réalisé au moyen d'un système d'interview téléphonique assistée par ordinateur (ITAO) et d'un système d'appel aléatoire. Les données ont été pondérées de manière à être représentatives de tous les adultes de 18 ans et plus à l'échelle du pays, et elles ont été équilibrées pour correspondre aux données de Statistique Canada les plus récentes en ce qui concerne le sexe, l'âge, le revenu et la région de résidence ${ }^{12}$. Les proportions et les moyennes ont été comparées statistiquement en utilisant un seuil de signification de $5 \%(p<0,05)$. L'IMC des répondants a été calculé en fonction de la taille et du poids autodéclarés et les répondants ont été classés dans les catégories suivantes : poids insuffisant $\left(<18,5 \mathrm{~kg} / \mathrm{m}^{2}\right)$, poids normal $\left(18,5-24,9 \mathrm{~kg} / \mathrm{m}^{2}\right)$, embonpoint $\left(25-29,9 \mathrm{~kg} / \mathrm{m}^{2}\right)$ et obésité $\left(\geq 30 \mathrm{~kg} / \mathrm{m}^{2}\right)$.
Par ailleurs, deux autres catégories ont été créées, soit IMC de $27 \mathrm{~kg} / \mathrm{m}^{2}$ ou plus avec états comorbides et IMC de $30 \mathrm{~kg} / \mathrm{m}^{2}$ ou plus sans états comorbides. Ces deux catégories supplémentaires correspondent aux seuils établis dans les Lignes directrices ${ }^{10}$. Comme le sondage portait sur les expériences de prise en charge du poids en relation avec l'embonpoint et l'obésité, les données sur le nombre négligeable de répondants présentant un poids insuffisant $(n=52)$ n’ont pas été prises en compte séparément dans notre analyse.

\section{Résultats}

\section{Caractéristiques de l'échantillon}

Au total, 2004 répondants au sondage ont été inclus dans l'analyse. De ce nombre, $48 \%$ étaient des hommes ( $\mathrm{n}=970$ ) et $52 \%$ des femmes ( $\mathrm{n}=1034) ; 78 \%$ ont été interviewés en anglais ( $\mathrm{n}=1557)$ et $22 \%$ en français ( $n=447)$. L'âge moyen des répondants était de 47 ans (écart-type de 16,4), $28 \%$ étaient de jeunes adultes (18-34 ans, $n=559$ ), 39 \% d'âge moyen (35-54 ans, $\mathrm{n}=788$ ) et $32 \%$ plus âgés (55 ans et plus, $n=649$ ). Sept répondants ont refusé de dévoiler leur âge. L'échantillon était composé de $81 \%$ de citadins ( $n=1615)$ et de $19 \%$ de ruraux $(n=388)$. L'IMC moyen était de $26,5 \mathrm{~kg} / \mathrm{m}^{2}$ $\left(27,3 \mathrm{~kg} / \mathrm{m}^{2}\right.$ pour les hommes et $25,7 \mathrm{~kg} / \mathrm{m}^{2}$ pour les femmes); $32 \%$ des répondants ont été classés comme ayant de l'embonpoint ( $\mathrm{n}=651$ ) et $21 \%$ comme étant obèses ( $\mathrm{n}=411$ ). Pourtant, $44 \%$ des répondants ont déclaré que leur poids était « convenable » $(\mathrm{n}=874), 37 \%$ ont dit avoir un léger embonpoint ( $n=734)$, et seulement $3 \%$ se sont décrits comme obèses $(n=51)$. Cette tendance était générale, sans égard au sexe ou à l'âge. Parmi les personnes ayant de l'embonpoint, $38 \%$ jugeaient leur poids " convenable " ( $\mathrm{n}=248 / 651)$; chez les personnes obèses, cette proportion était de $4 \%(n=18 / 411)$ et, chez les personnes ayant un poids normal, elle était de $73 \%$ (n = 567/779). Sur les personnes interrogées, $41 \%$ ont dit n'avoir jamais tenté de perdre du poids ( $\mathrm{n}=818$ ). Dans les 12 mois précédant le sondage, davantage de femmes (52 \%) que d'hommes (37\%) avaient tenté de perdre du poids, cet écart augmentant 
avec l'âge. En outre, une tendance similaire a été observée chez les personnes qui ont tenté de perdre du poids plus de 12 mois avant le sondage.

\section{Objectif 1 : Expérience de prise en charge du poids des Canadiens}

Le sondage visait à déterminer si les adultes canadiens ayant de l'embonpoint ou obèses avaient cherché à obtenir de l'aide auprès d'un médecin de famille ou d'un autre professionnel de la santé relativement à la prise en charge de leur poids. La majorité des personnes ayant de l'embonpoint ( $64 \%, \mathrm{n}=418 / 651$ ) et des personnes obèses ( $88 \%, n=363 / 411$ ) ont admis avoir déjà tenté de perdre du poids, la plupart au cours des 12 mois précédant le sondage; les taux étaient similaires chez les personnes avec un IMC de $30 \mathrm{~kg} / \mathrm{m}^{2}$ ou plus sans états comorbides $(85 \%$ ont tenté de perdre du poids, $\mathrm{n}=188 / 221$ ) et chez celles avec un IMC de $27 \mathrm{~kg} / \mathrm{m}^{2}$ ou plus avec états comorbides $(86 \%$ ont tenté de perdre du poids, $n=253 / 293)$. L'écart entre le nombre de personnes ayant de l'embonpoint qui ont tenté de perdre du poids au cours des 12 mois précédant le sondage et le nombre de répondants ayant un poids normal qui ont fait la même démarche était significatif; le même constat s'applique à la comparaison entre les personnes obèses et les répondants ayant de l'embonpoint ou un poids normal. Le tableau 1 présente les caractéristiques des expériences de prise en charge de leur poids par les répondants.

La plupart des répondants ( $85 \%, \mathrm{n}=1711$ ) ont consulté un médecin (pour une affection quelconque, pas nécessairement en raison de leur poids) au moins une fois au cours des 12 mois précédant le sondage. Les proportions étaient globalement similaires pour tous les groupes, mais certaines tendances ont pu être observées. En effet, davantage de femmes (90\%) que d'hommes (80 \%) ont vu un médecin au cours de cette période, et le pourcentage de consultation était plus élevé au sein du groupe le plus âgé (55 ans et plus, $91 \%$ ) qu'au sein des deux groupes d'âge plus jeunes (18-34 ans ainsi que $35-54$ ans, $83 \%$ ). En outre, plus
l'IMC était élevé, plus le nombre de visites chez le médecin était élevé également. Le taux de consultation le plus élevé a été observé chez les répondants avec un IMC de $27 \mathrm{~kg} / \mathrm{m}^{2}$ ou plus avec états comorbides (98\%, $\mathrm{n}=289 / 293)$. Nous avons calculé le nombre moyen de visites par répondant $(2,0)$ en nous fondant sur l'ensemble des répondants, incluant ceux qui ont déclaré ne pas avoir consulté de médecin dans les 12 mois précédant le sondage.

Au total, $67 \%$ des répondants ont déclaré ne pas avoir spécifiquement abordé la question de la perte de poids avec leur professionnel de la santé ( $\mathrm{n}=1339$ ) et, parmi ceux qui l'ont fait (n = 665), $72 \%$ ont consulté leur médecin de famille ( $\mathrm{n}=482$ ), $24 \%$ un diététiste ( $\mathrm{n}=158), 16 \%$ un nutritionniste ( $\mathrm{n}=108), 16 \%$ une infirmière ou une infirmière praticienne $(\mathrm{n}=107)$ et $9 \%$ un pharmacien $(n=59)$. Le pourcentage total est supérieur à $100 \%$ étant donné que certains répondants ont consulté plus d'un professionnel de la

TABLEAU 1

Caractéristiques des visites chez le médecin et des tentatives de perte de poids des répondants au sondage

\begin{tabular}{|c|c|c|c|c|c|c|c|}
\hline \multirow{2}{*}{$\begin{array}{l}\text { Catégories de } \\
\text { répondants }\end{array}$} & \multirow{2}{*}{$\begin{array}{c}\text { Taille de } \\
\text { l'échantillon } \\
\mathbf{n}\end{array}$} & \multicolumn{3}{|c|}{ Visites chez le médecin } & \multicolumn{3}{|c|}{ Tentatives de perte de poids } \\
\hline & & $\begin{array}{l}\text { A vu un médecin } \\
\text { au cours des } \\
12 \text { derniers mois } \\
\text { n (\%) }\end{array}$ & $\begin{array}{c}\text { Nombre moyen } \\
\text { de visites chez le } \\
\text { médecin }^{b}\end{array}$ & $\begin{array}{l}\text { Est suivi par un } \\
\text { médecin pour } \\
\text { une affection } \\
\text { n (\%) }\end{array}$ & $\begin{array}{c}\text { Dernière } \\
\text { tentative de perte } \\
\text { de poids au } \\
\text { cours des } \\
12 \text { derniers mois } \\
n(\%)\end{array}$ & $\begin{array}{c}\text { Dernière } \\
\text { tentative de perte } \\
\text { de poids il y a } \\
\text { plus de } 12 \text { mois } \\
n(\%)\end{array}$ & $\begin{array}{c}\text { N'a jamais } \\
\text { tenté de perdre } \\
\text { du poids } \\
\text { n (\%) }\end{array}$ \\
\hline Tous $^{\mathrm{c}}$ & 2004 & $1711 \quad(85 \%)$ & 2,86 & $808 \quad(40 \%)$ & $897 \quad(45 \%)$ & $283(14 \%)$ & $818 \quad(41 \%)$ \\
\hline $\begin{array}{l}\text { Poids normal } \\
\left(\mathrm{IMC} 18,5-24,9 \mathrm{~kg} / \mathrm{m}^{2}\right)\end{array}$ & 779 & $638 \quad(82 \%)$ & 2,58 & $247 \quad(32 \%)$ & $214 \quad(27 \%)$ & $100 \quad(13 \%)$ & $462^{\mathrm{d}, \mathrm{e}}(59 \%)$ \\
\hline $\begin{array}{l}\text { Embonpoint } \\
\left(\text { IMC } 25,0-29,9 \mathrm{~kg} / \mathrm{m}^{2}\right)\end{array}$ & 651 & $559 \quad(86 \%)$ & 2,65 & $272^{f} \quad(42 \%)$ & $326^{f} \quad(50 \%)$ & $92 \quad(14 \%)$ & $230^{\mathrm{e}} \quad(35 \%)$ \\
\hline $\begin{array}{l}\text { Obésité } \\
(I M C \geq 30,0 \text { kg/m²) }\end{array}$ & 411 & $364^{f} \quad(89 \%)$ & 3,47 & $233^{\mathrm{d}, \mathrm{f}}(57 \%)$ & $290^{\mathrm{d}, \mathrm{f}}(71 \%)$ & $73^{f} \quad(18 \%)$ & $41 \quad(10 \%)$ \\
\hline $\begin{array}{l}\text { IMC } \geq 30,0 \mathrm{~kg} / \mathrm{m}^{2} \text { sans } \\
\text { états comorbides }\end{array}$ & 221 & $178 \quad(81 \%)$ & 2,82 & $43 \quad(19 \%)$ & $139 \quad(63 \%)$ & $49 \quad(22 \%)$ & $32 \quad(15 \%)$ \\
\hline $\begin{array}{l}\text { IMC } \geq 27,0 \mathrm{~kg} / \mathrm{m}^{2} \text { avec } \\
\text { états comorbides }\end{array}$ & 293 & $289 \quad(98 \%)$ & 3,96 & $293(100 \%)$ & $210 \quad(71 \%)$ & $43 \quad(15 \%)$ & $40 \quad(14 \%)$ \\
\hline \multicolumn{8}{|c|}{ Abréviations : IMC, indice de masse corporelle; $\mathrm{n}$, taille de l'échantillon; $p$, valeur $p$. } \\
\hline \multicolumn{8}{|c|}{ Basé sur tous les répondants, y compris ceux ayant indiqué ne pas avoir consulté un médecin au cours des 12 derniers mois. Nombre médian de visites = 2,0. } \\
\hline \multicolumn{8}{|c|}{$\begin{array}{l}\text { c Les données sur les répondants présentant un poids insuffisant }(n=52) \text { et les répondants ne connaissant pas leur poids ou ayant refusé de fournir celui-ci ( } \mathrm{n}=111) \text { sont incluses } \\
\text { dans ces totaux, mais ne sont pas présentées ailleurs dans ce tableau. }\end{array}$} \\
\hline
\end{tabular}


santé. Les taux de consultation étaient plus élevés chez les femmes que chez les hommes et ils augmentaient avec l'âge ainsi qu'avec l'IMC.

Seulement $15 \%$ des répondants avaient interrogé leur médecin de famille au sujet de la perte de poids $(\mathrm{n}=295)$ au cours des 12 mois précédant le sondage, les femmes (17 \%) ayant été légèrement plus nombreuses à le faire que les hommes (13 \%). Là aussi, la proportion de ceux ayant posé des questions à leur médecin au sujet de la perte de poids augmentait à la fois avec l'âge, passant de $10 \%$ (pour les 18-24 ans) à $18 \%$ ( pour les 55 ans et plus), et avec l'IMC, passant de $24 \%$ pour les personnes faisant de l'embonpoint à $56 \%$ pour les personnes obèses. Environ la moitié de ceux qui avaient posé des questions à propos de la perte de poids (13 \% des personnes faisant de l'embonpoint et $35 \%$ des personnes obèses) l'avaient fait au cours des 12 mois précédant le sondage.

Sur l'ensemble des répondants, $40 \%$ ont déclaré recevoir des soins d'un médecin pour une affection quelconque ( $\mathrm{n}=808 / 2$ 004). Davantage de femmes (44\%) que d'hommes (37\%) ont affirmé être dans une telle situation, ce taux augmentant avec l'âge, allant de $19 \%$ chez les jeunes adultes (18-34 ans) à $66 \%$ chez les adultes plus âgés (55 ans et plus).
Dans un même ordre d'idée, on a observé une augmentation reliée à l'âge du pourcentage de répondants recevant des soins pour des états comorbides liés au poids, dans une fourchette allant de $6 \%$ à $56 \%$. Environ la moitié des répondants faisant de l'embonpoint ( $42 \%, \mathrm{n}=272 / 651$ ) ou obèses $(57 \%, \mathrm{n}=233 / 411)$ étaient suivis par un médecin pour une affection quelconque, et respectivement $30 \%$ ( $n=193)$ et $46 \%(n=190)$ pour des états comorbides spécifiques liés au poids. Le tableau 2 indique le taux de prévalence autodéclaré des affections médicales les plus courantes.

Il est intéressant de souligner que seulement $30 \%$ des répondants faisant de l'embonpoint ou obèses ont affirmé que leur médecin leur avait conseillé de perdre du poids sans qu'ils aient eux-mêmes abordé la question ( $n=320 / 1$ 062). De ce nombre, $19 \%$ ( $n=197 / 1$ 062) estimaient que ce conseil visait l'amélioration de leur santé en général, et $12 \%$, qu'il visait l'amélioration du traitement d'une autre affection médicale. La tendance était similaire chez les hommes et chez les femmes. Cependant, les personnes d'âge moyen (35-54 ans, $21 \%$ ) et les adultes les plus âgés (55 ans et plus, $24 \%$ ) ont reçu davantage de conseils que les jeunes adultes (18-34 ans; $11 \%$ ), la proportion augmentant en fonction de l'IMC (normal, $5 \%$; embonpoint, $18 \%$; obésité, $49 \%$ ),

TABLEAU 2

Taux auto-déclarés de prévalence d'affections médicales courantes par catégorie d'IMC

\begin{tabular}{|c|c|c|c|c|}
\hline \multirow{2}{*}{ Affection médicale } & \multicolumn{4}{|c|}{ Répondantsa , n (\%) } \\
\hline & $\begin{array}{c}\text { Tous } \\
(n=2004)\end{array}$ & $\begin{array}{l}\text { Poids normal } \\
\quad(n=779)\end{array}$ & $\begin{array}{l}\text { Embonpoint }{ }^{c} \\
\quad(n=651)\end{array}$ & $\begin{array}{l}\text { Obésitéd }^{d} \\
(n=411)\end{array}$ \\
\hline Hypertension artérielle & $301(15)$ & 67 (9) & $99(15)^{e}$ & $111(27)^{\mathrm{e}, \mathrm{f}}$ \\
\hline Taux élevé de cholestérol & $211(11)$ & $48 \quad(6)$ & $78(12)^{e}$ & $74(18)^{e, f}$ \\
\hline $\begin{array}{l}\text { Maladie cardiovasculaire/ } \\
\text { cardiaque }\end{array}$ & $99 \quad(5)$ & $23(3)$ & $35(5)^{e}$ & $33(8)^{e}$ \\
\hline Diabète & $146(7)$ & 35 (5) & $51(8)^{e}$ & $55(13)^{e, f}$ \\
\hline Arthrose & 134 (7) & $36 \quad(5)$ & $43(7)$ & $51(12)^{e, f}$ \\
\hline Autres & $369(18)$ & $134(17)$ & $116(18)$ & $88(22)$ \\
\hline
\end{tabular}

Abréviations : IMC, indice de masse corporelle; $\mathrm{n}$, taille de l'échantillon; $p$, valeur $\mathrm{p}$.

${ }^{a}$ Données pondérées.

${ }^{\mathrm{b}}$ IMC $=18,5-24,9 \mathrm{~kg} / \mathrm{m}^{2}$.

${ }^{\mathrm{C}}$ IMC $=25,0-29,9 \mathrm{~kg} / \mathrm{m}^{2}$.

${ }^{\mathrm{d}}$ IMC $\geq 30,0 \mathrm{~kg} / \mathrm{m} 2$.

${ }^{\mathrm{e}}$ Significativement différent par rapport aux répondants dont le poids est normal $(p<0,05)$.

f Significativement différent par rapport aux répondants faisant de l'embonpoint $(p<0,05)$. les personnes ayant une IMC de $27 \mathrm{~kg} / \mathrm{m}^{2}$ ou plus avec états comorbides étant celles qui avaient reçu le plus de conseils (54\%). Les répondants obèses ont compris que les conseils quant à la perte de poids visaient l'amélioration de leur santé en général (30 \%) et l'amélioration du traitement d'une autre affection médicale (19 \%). Chez les personnes avec un IMC de $27 \mathrm{~kg} / \mathrm{m}^{2}$ ou plus avec états comorbides, cette proportion était respectivement de $28 \%$ et de $26 \%$.

Sur les 383 personnes faisant de l'embonpoint ou obèses qui ont demandé de l'aide à un professionnel de la santé pour perdre du poids, 15 (4\%) ont indiqué n’avoir reçu aucun conseil, 261 (68 \%) ont affirmé avoir reçu des conseils en matière d'alimentation, 237 (62\%) ont dit avoir reçu des conseils en matière d'exercice physique, 47 (12 \%) ont été invités à participer à un programme de perte de poids, à visiter une clinique d'amaigrissement ou à prendre des substituts de repas ou des suppléments, et 15 (4\%) ont obtenu une ordonnance de médicament antiobésité. Sur les 230 répondants obèses ayant demandé de l'aide pour perdre du poids, 10 (4\%) ont déclaré avoir reçu des conseils sur les possibilités de chirurgie, ce qui a été le cas pour un répondant ayant de l'embonpoint.

\section{Objectif 2 : Déterminer si les pratiques de prise en charge du poids des médecins de famille étaient conformes aux Lignes directrices canadiennes}

Nous avons voulu déterminer si les médecins de famille des répondants au sondage faisaient appel aux pratiques de prise en charge du poids recommandées dans les Lignes directrices ${ }^{10}$. Le tableau 3 indique le nombre de répondants dont la circonférence de la taille a été mesurée dans les 12 mois précédant le sondage et qui, pendant cette période, ont subi un test de glycémie et ont fait vérifier leur pression artérielle. Seulement $14 \%$ des répondants ont affirmé qu'un médecin avait mesuré la circonférence de leur taille ( $n=285 / 2$ 004); cette proportion était légèrement plus importante chez les hommes (17 \%) que chez les femmes (12\%) et augmentait avec l'âge, passant de $11 \%$ chez les jeunes adultes (18-34 ans) 
TABLEAU 3

Pratiques de prise en charge du poids par les médecins de famille par rapport à trois recommandations des Lignes directrices canadiennes de pratique clinique de 2006, selon les déclarations des répondants

\begin{tabular}{|c|c|c|c|c|c|c|c|c|c|c|}
\hline \multirow{3}{*}{$\begin{array}{l}\text { Catégories de } \\
\text { répondants }\end{array}$} & \multirow{3}{*}{$\begin{array}{c}\text { Taille de } \\
\text { l'échantillon } \\
\text { n }\end{array}$} & \multicolumn{9}{|c|}{ Pratiques respectant les Lignes directrices de pratique clinique ${ }^{\mathrm{a}}$, n (\%) } \\
\hline & & \multicolumn{3}{|c|}{ Circonférence de la taille } & \multicolumn{3}{|c|}{ Pression artérielle } & \multicolumn{3}{|c|}{ Test de dépistage du diabète } \\
\hline & & $\begin{array}{l}\text { Au cours } \\
\text { des } \\
12 \text { derniers } \\
\text { mois }\end{array}$ & $\begin{array}{c}\text { Pas au } \\
\text { cours des } \\
12 \text { derniers } \\
\text { mois }\end{array}$ & $\begin{array}{c}\text { Ne sait } \\
\text { pas }\end{array}$ & $\begin{array}{l}\text { Au cours } \\
\text { des } \\
12 \text { derniers } \\
\text { mois }\end{array}$ & $\begin{array}{l}\text { Il y a plus } \\
\text { de } 12 \text { mois }\end{array}$ & Jamais & $\begin{array}{l}\text { Au cours } \\
\text { des } \\
12 \text { derniers } \\
\text { mois }\end{array}$ & $\begin{array}{l}\text { Il y a plus de } \\
12 \text { mois }\end{array}$ & Jamais \\
\hline Tous & 2004 & $285(14)$ & $1700(85)$ & $19(1)$ & $1603(80)$ & $313(16)$ & $80(4)$ & $723(36)$ & $443(22)$ & $812(41)$ \\
\hline $\begin{array}{l}\text { Poids normal } \\
(\text { IMC 18,5-24,9 kg/m²) }\end{array}$ & 779 & $100(13)$ & $672(86)$ & $7(1)$ & $581(75)$ & $150(19)^{b, c}$ & $43(5)^{b, c}$ & $221(28)$ & $166(21)$ & $382(49)^{b, c}$ \\
\hline $\begin{array}{l}\text { Embonpoint } \\
\left(25,0-29,9 \mathrm{~kg} / \mathrm{m}^{2}\right)\end{array}$ & 651 & $92(14)$ & $554(85)$ & $6(1)$ & $536(82)^{d}$ & 93 (14) & $20(3)^{c}$ & $235(36)^{d}$ & $151(23)$ & 254 (39) \\
\hline $\begin{array}{l}\text { Obésité } \\
\left(\geq 30,0 \mathrm{~kg} / \mathrm{m}^{2}\right)\end{array}$ & 411 & $73(18)^{d}$ & $335(82)$ & $3(1)$ & $358(87)^{d}$ & $50(12)$ & $3(1)$ & $206(50)^{b, d}$ & $98(24)$ & 104 (25) \\
\hline $\begin{array}{l}\mathrm{IMC} \geq 30,0 \mathrm{~kg} / \mathrm{m}^{2} \text { sans } \\
\text { états comorbides }\end{array}$ & 221 & $32(14)$ & $187(85)$ & $1(0)$ & 173 (78) & $45(20)$ & $3(1)$ & 75 (34) & $61(28)$ & 82 (37) \\
\hline $\begin{array}{l}\text { IMC } \geq 27,0 \mathrm{~kg} / \mathrm{m}^{2} \text { avec } \\
\text { états comorbides }\end{array}$ & 293 & $61(21)$ & 230 (79) & $2(1)$ & 283 (97) & $10 \quad(3)$ & $0(0)$ & $186(63)$ & $62(21)$ & 44 (15) \\
\hline
\end{tabular}

Abréviations : IMC, indice de masse corporelle; $\mathrm{n}$, taille de l'échantillon; $p$, valeur $p$.

a Données pondérées.

${ }^{b}$ Significativement différent par rapport aux répondants ayant de l'embonpoint $(p<0,05)$.

c Significativement différent par rapport aux répondants obèses $(p<0,05)$.

${ }^{\mathrm{d}}$ Significativement différent par rapport aux répondants dont le poids est normal $(p<0,05)$.

à respectivement $14 \%$ chez les personnes d'âge moyen (35-54 ans) et $18 \%$ chez les adultes plus âgés (55 ans et plus). La mesure de la circonférence de la taille dans l'année précédant le sondage n'a été que légèrement influencée par l'IMC (poids normal $13 \%$; embonpoint $14 \%$; obésité $18 \%$ ) et n’a été que légèrement plus fréquente chez les personnes avec un IMC de $27 \mathrm{~kg} / \mathrm{m}^{2}$ avec états comorbides (21 \%) par rapport aux personnes obèses, mais la différence entre les personnes dont le poids est normal et les personnes obèses était significative. Toutefois, la circonférence de la taille de $84 \%$ des répondants ayant de l'embonpoint ou obèses n'avait pas été mesurée dans l'année précédant le sondage ( $\mathrm{n}=889 / 1$ 062).

En ce qui concerne la mesure de la pression artérielle dans les 12 mois précédant le sondage, une tendance a été observée en fonction du sexe et de l'âge. Les femmes ont affirmé dans une proportion légèrement supérieure $(85 \%$, contre $75 \%$ chez les hommes) que leur pression artérielle avait été mesurée; cette proportion était de $69 \%$ chez les jeunes adultes (18-34 ans) et de $90 \%$ chez les adultes plus âgés (55 ans et plus). Cette tendance augmentait aussi en fonction de l'IMC. Les femmes ont été légèrement plus nombreuses (65\%) que les hommes (51 \%) à déclarer avoir subi un test de dépistage du diabète au cours des 12 mois précédant le sondage, et cette proportion augmentait également avec l'âge (de $40 \%$ chez les 18-34 ans à $74 \%$ chez les 55 ans et plus) et l'IMC. Les écarts relatifs à la mesure de la pression artérielle et au test de dépistage du diabète au cours des 12 mois précédant le sondage étaient significatifs entre les répondants ayant de l'embonpoint ou obèses et les répondants présentant un poids normal.

\section{Discussion}

Ce sondage donne un aperçu des expériences actuelles de prise en charge du poids des Canadiens et met en lumière les pratiques de prise en charge de leur poids adoptées par leur médecin par rapport à trois recommandations des Lignes directrices canadiennes de 2006. Sur l'ensemble des répondants au sondage, $53 \%$ ont déclaré avoir de l'embonpoint (33\%) ou être obèse (20\%), ce qui est légèrement inférieur aux données canadiennes publiées (embonpoint, $36 \%$ et obésité, $23 \%)^{1}$, ce qui est probablement dû au fait que des données autodéclarées ont été utilisées en ce qui concerne le poids et la taille (en règle générale, les répondants sous-estiment leur poids et surestiment leur taille) ${ }^{13}$. Il est aussi intéressant de noter que $40 \%$ des répondants faisant de l'embonpoint ou obèses ont considéré que leur poids était " convenable ». Ce phénomène a été observé dans le cadre d'autres études et pourrait être dû à la normalisation de la prise excessive de poids à mesure que les taux d'obésité augmentent ${ }^{14,15}$. Cela entraîne des répercussions sur les fournisseurs de soins de santé, qui auront peut-être à sensibiliser leurs patients au sujet des risques pour la santé associés à l'embonpoint et à l'obésité avant de leur fournir des conseils en matière de prise en charge du poids.

Même si plus de la moitié des répondants au sondage ont affirmé avoir de l'embonpoint ou être obèse, un nombre étonnamment peu élevé d'entre eux a reçu ou demandé à recevoir des conseils sur la perte de poids de la part d'un professionnel de la santé. La majorité a déclaré avoir consulté 
son médecin dans les 12 mois précédant le sondage - cette proportion étant beaucoup plus élevée chez les répondants obèses - et environ les trois quarts des adultes canadiens sondés faisant de l'embonpoint ou obèses avaient déjà tenté de perdre du poids, dont $58 \%$ au cours des 12 mois précédant le sondage. Pourtant, seulement $21 \%$ des répondants faisant de l'embonpoint ont déclaré avoir demandé de l'aide à leur médecin dans l'année précédant le sondage, ce qui donne à penser que la majorité d'entre eux estimaient que la responsabilité de la perte de poids leur incombait personnellement. Qui plus est, moins du tiers des répondants faisant de l'embonpoint/obèses ont reçu des conseils de leur médecin sur la perte de poids (sans en avoir fait spécifiquement la demande), illustrant là aussi que l'idée selon laquelle l'obésité est un problème de responsabilité personnelle plutôt qu'un problème médical est largement répandue dans la société ${ }^{15}$. Par conséquent, il y a de bonnes chances que la majorité des tentatives de perte de poids déclarées aient été entreprises à l'initiative des répondants plutôt qu'à la suite de conseils formulés par un professionnel de la santé, les régimes amaigrissants et l'exercice physique étant les deux méthodes ayant été les plus fréquemment utilisées. Les répondants ont aussi cherché à obtenir du soutien auprès de diététistes, d'infirmières et de pharmaciens, mettant en évidence le rôle de ces différents professionnels de la santé dans la prise en charge du poids.

En règle générale, les répondants faisant de l'embonpoint ou obèses qui ont interrogé leur médecin au sujet de la perte de poids ont reçu des conseils sur les régimes alimentaires et l'exercice, seulement $4 \%$ des répondants obèses ayant affirmé avoir reçu des conseils sur les différentes solutions chirurgicales. Cette dernière statistique est particulièrement digne d'intérêt, compte tenu du rôle prometteur qu'occupera la chirurgie comme principale option de traitement à long terme pour les personnes souffrant d'obésité sévère ${ }^{16,17}$. Bien que le critère pour envisager la chirurgie soit un IMC de $40 \mathrm{~kg} / \mathrm{m}^{2}$ ou plus - ou de $35 \mathrm{~kg} / \mathrm{m}^{2}$ ou plus avec états comorbides - et quoique les personnes interrogées aient été jugées obèses lorsqu'elles déclaraient avoir un IMC de $30 \mathrm{~kg} / \mathrm{m}^{2}$ ou plus, il faudrait inclure les interventions chirurgicales dans la liste des solutions pour la prise en charge du poids comme le font les Lignes directrices de pratique clinique existantes ${ }^{10}$.

Au Canada, les médecins disposent de nombreuses occasions pour dépister les états comorbides et ils le font régulièrement : $84 \%$ des répondants faisant de l'embonpoint ou obèses ont fait vérifier leur pression artérielle dans l'année précédant le sondage, ce qui cadre avec les recommandations de 2009 du Programme éducatif canadien de l'hypertension ${ }^{18}$. Dans un même ordre d'idée, selon les lignes directrices de pratique clinique de 2008 de l'Association canadienne du diabète, les personnes âgées de 40 ans et plus devraient se soumettre à un test de dépistage du diabète de type 2 (épreuve d'hyperglycémie plasmatique à jeun) tous les trois ans, tandis que les personnes présentant des facteurs de risque supplémentaires devraient passer un test de dépistage plus tôt ou plus souvent ${ }^{19}$. Le sondage a révélé que $66 \%$ des Canadiens faisant de l'embonpoint ou obèses s'étaient déjà soumis à un test de dépistage du diabète. Ces résultats sont encourageants, mais il faut quand même s'attaquer à l'obésité plus tôt dans le processus de prise de poids pour prévenir l'apparition de maladies chroniques ${ }^{1}$. Les réponses de la majorité des répondants indiquent que les médecins respectaient les principales recommandations en ce qui concerne le dépistage de l'hypertension et du diabète, mais qu'ils ne suivaient pas les recommandations pour ce qui est de l'évaluation de l'obésité au moyen de la mesure de la circonférence de la taille $\mathrm{1}^{10,18,19}$.

Malgré l'ampleur de l'épidémie d’obésité, les résultats du sondage révèlent, de façon surprenante, que peu de patients ayant de l'embonpoint ou obèses ont reçu des conseils relativement à la prise en charge de leur poids, ce qui laisse croire que le système de santé ne fournit pas de services convenables à cet égard ${ }^{9}$. Cette situation s'observe en dépit du fait que l'obésité est de plus en plus répandue ${ }^{3}$ et de plus en plus reconnue comme une maladie à proprement parler ${ }^{8}$. Une des raisons pour lesquelles les médecins sont plus susceptibles de traiter l'hypertension et le diabète que l'obésité est que les deux premières sont des maladies reconnues, tandis que l'obésité est encore uniquement considérée comme un facteur de risque de maladie ${ }^{20}$. Quoique les spécialistes de l'obésité aient réfuté cette conception ${ }^{21}$, il reste que l'obésité n'est généralement pas considérée comme une maladie, ce qui peut constituer un obstacle à l'amélioration de sa prise en charge. En outre, on considère en général dans la société l'obésité comme un état causé par l'absence de volonté, et les personnes faisant de l'embonpoint ou obèses comme manquant de volonté, peu soignées et paresseuses ${ }^{22}$. Les professionnels de la santé partagent souvent ce point de vue, ce qui peut avoir une incidence sur la façon dont ils abordent la question de la prise en charge du poids avec les personnes faisant de l'embonpoint et obèses ${ }^{22}$. Ainsi, si l'on s'attaque à la question des préjugés et de la stigmatisation à l'égard de l'embonpoint et de l'obésité, on pourrait améliorer à la fois la manière dont les conseils de prise en charge du poids sont offerts dans le système de santé et la manière dont ils sont reçus ${ }^{22}$.

\section{Limites}

Les limites de notre étude sont liées au fait que celle-ci repose sur des données autodéclarées. Comme il a été mentionné précédemment, les répondants sousestiment leur poids et surestiment leur taille, entraînant une sous-estimation de la prévalence de l'obésité ${ }^{13}$. De plus, le comportement des médecins n'a pas fait l'objet d'une évaluation objective, même si ce sondage sert en fait de mesure substitutive, en donnant un aperçu du comportement des professionnels de la santé tel qu'il a été observé par leurs patients. Les répondants n'ont pas été interrogés au sujet de leur perception de la qualité des soins de santé qu'ils ont reçus ou des interactions avec leur fournisseur de soins de santé, notamment en ce qui concerne les préjugés dont ils auraient pu être victimes en raison de leur poids $^{22}$. Comme les conseils d'un médecin sont de puissants éléments de motivation par rapport à la perte de poids ${ }^{23}$, il est préoccupant de constater que les médecins n'encouragent pas systématiquement leurs patients faisant de l'embonpoint ou obèses à perdre du poids. Peu d'études ont été menées sur les solutions à adopter pour que les professionnels de la santé changent 
la façon dont ils voient et prennent en charge l'obésité, comme l'indique le nombre peu élevé d'études recensées dans le cadre d'un examen systématique de Cochrane sur ce sujet ${ }^{24}$. Toutefois, les données probantes disponibles indiquent que les médecins et les autres professionnels de la santé jouent un rôle dans la prise en charge de l'obésité24, ce qui donne de l'espoir quant à la prise en charge de cette condition dans le futur. Les médecins canadiens ont exprimé le besoin de consacrer plus de temps à leurs patients et de diminuer le nombre de patients qu'ils voient par heure ${ }^{25}$. En outre, ils sont prêts à orienter leurs patients vers d'autres professionnels de la santé, par exemple des diététistes, mais ils font part dans le même temps de leurs préoccupations quant à la rapidité avec laquelle leurs patients auront accès à ces professionnels et ils estiment qu'un processus d'aiguillage plus rapide est nécessaire ${ }^{25}$. Il faut, de toute évidence, que les lignes directrices actuelles sur l'obésité soient mises en œuvre et évaluées de façon plus efficace, et que cette mise en œuvre soit appuyée par un plus grand nombre de ressources, particulièrement dans les points de service ${ }^{26}$. Enfin, et il s'agit peut-être du point le plus important, il faut absolument venir à bout des préjugés et de la stigmatisation liés à l'obésité dans la société, y compris chez les professionnels de la santé22, et reconnaître que les personnes obèses présentent souvent d'autres problèmes susceptibles de les empêcher de perdre du poids, en particulier des problèmes de santé mentale, des douleurs chroniques et des obstacles familiaux ou sociaux ${ }^{27}$. Bien que ces éléments n'aient pas été mesurés par le sondage, on sait qu'ils ont une incidence sur la capacité à prendre son poids en charge et à maintenir ses efforts ${ }^{22,26}$. Il est nécessaire que les professionnels de la santé connaissent ces défis et les relèvent pour améliorer les pratiques de prise en charge de l'obésité au sein du système de santé canadien.

\section{Remerciements}

Ce sondage a été réalisé grâce à l'octroi d'une subvention d'éducation sans conditions au Réseau canadien en obésité par Glaxo-Smith Kline.

\section{Références}

1. Tjepkema M. Obésité chez les adultes. Rapp. santé. 2006;17(3):9-25.

2. World Health Organization. Obesity: preventing and managing the global epidemic - report of a WHO consultation. Geneva (CH): WHO; 1997 Jun. Report No.: WHO/NUT/NCD/98.1.

3. Anis AH, Zhang W, Bansback N, Guh DP, Amarsi Z, Birmingham CL. Obesity and overweight in Canada: an updated costof-illness study. Obes Rev. 2010;11(1):31-40.

4. Jia H, Lubetkin EI. Trends in qualityadjusted life-years lost contributed by smoking and obesity. Am J Prev Med. 2010;38(2):138-144.

5. Katzmarzyk PT, Mason C. Prevalence of class I, II and III obesity in Canada. CMAJ. 2006;174(2):156-7.

6. Block JP, DeSalvo KB, Fisher WP. Are physicians equipped to address the obesity epidemic? Knowledge and attitudes of internal medicine residents. Prev Med. 2003;36(6):669-75.

7. Epstein L, Ogden J. A qualitative study of GPs' views of treating obesity. Br J Gen Pract. 2005;55(519):750-4.

8. Jackson JE, Doescher MP, Saver BG, Hart LG. Trends in professional advice to lose weight among obese adults, 1994 to 2000. J Gen Intern Med. 2005;20(9):814-8.

9. Padwal RS, Damjanovic S, Schulze KM, Lewanczuk RZ, Lau DC, Sharma AM. Canadian physicians' use of antiobesity drugs and their referral patterns to weight management programs or providers: the SOCCER study. J Obes. 2011;686521. Epub 2010 Nov 21. doi: 10.1155/2011/686521

10. Lau DC, Douketis JD, Morrison KM, Hramiak IM, Sharma AM, Ur E et collab. Lignes directrices canadiennes de 2006 sur la prise en charge et la prévention de l'obésité chez les adultes et les enfants [sommaire] [Internet]. JAMC. 2007 [consultation déc. 2010];176(8):1103-6. Consultable à la page : http://www.cmaj.ca /content/176/8/SF1

11. McDonald SD. Management and prevention of obesity in adults and children. CMAJ. 2007;176(8):1109-10.
12. Annuaire du Canada 2008. Aperçu consultable à partir de la page : http: / / w w w 41 . st a t c a n.g c.c a / 2008 /ceb_r000_2008-fra.htm

13. Visscher TL, Viet AL, Kroesbergen HT, Seidell JC. Underreporting of BMI in adults and its effect on obesity prevalence estimations in the period 1998 to 2001. Obesity. 2006;14:2054-63.

14. Johnson F, Cooke L, Croker H, Wardle J. Changing perceptions of weight in Great Britain: comparison of two population surveys. BMJ. 2008;337(7761):a494. doi: 10.1136/bmj.a494

15. Pulvers KM, Kaur H, Nollen NL, Greiner KA, Befort CA, Hall S et collab. Comparison of body perceptions between obese primary care patients and physicians: implications for practice. Patient Educ Couns. 2008;73(1):73-81.

16. Buchwald H, Avidor Y, Braunwald E, Jensen MD, Pories W, Fahrbach K et collab. La chirurgie de l'obésité : revue systématique et méta-analyse. JAMA. 2004;292(14):546-559.

17. Livingston EH, Fink AS. Quality of life: cost and future of bariatric surgery. Arch Surg. 2003;138(4):383-8.

18. Canadian Hypertension Education Program. The 2008 Canadian Hypertension Education Program recommendations: the scientific summary - an annual update. Can J Cardiol. 2008;24(6):447-52.

19. Comité d'experts des Lignes directrices de pratique clinique de l'Association canadienne du diabète. Lignes directrices de pratique clinique 2008 de l'Association canadienne du diabète pour la prévention et le traitement du diabète au Canada. Can J Diabetes. 2008;32(suppl. 2):S1-S225.

20. Heshka S, Allison DB. Is obesity a disease? Int J Obes Relat Metab Disord. 2001;25:1401-4.

21. The Council of the Obesity Society. Obesity as a disease: The Obesity Society Council Resolution. Obesity. 2008;16(6):1151. doi:10.1038/oby.2008.246.

22. Puhl RM, Heuer CA. The stigma of obesity: a review and update. Obesity. 2009;17(5):941-64. doi:10.1038/oby.2008.636 
23. Loureiro ML, Nayga J,Rodolfo M. Obesity, weight loss, and physician's advice. Soc Sci Med. 2006 5;62(10):2458-68.

24. Flodgren G, Deane K, Dickinson HO, Kirk S, Alberti H, Beyer FR, et collab. Interventions to change the behaviour of health professionals and the organisation of care to promote weight reduction in overweight and obese people [Internet]. Cochrane DB Syst Rev. 2010 [consultation déc. 2010];(3). Consultable à partir de la page : www.mrw.interscience.wiley.com/cochrane /clsysrev/articles/CD000984/frame.html doi: 10.1002/14651858.CD000984.pub2

25. Moores DG, Wilson DR, Cave AJ, Woodhead Lyons SC, Donoff MG. Improving the quality and capacity of Canada's health services: primary care physician perspectives. Healthc Policy. 2007;3(2):1-17

26. Sharma AM, Kushner RF. A proposed clinical staging system for obesity. Int $\mathrm{J}$ Obes (Lond). 2009;33(3):289-95.

27. Mauro M, Taylor V, Wharton S, Sharma AM. Barriers to obesity treatment. Eur J Intern Med. 2008;19(3):173-80. 